\author{
Jean-Pierre Pelletier \\ Lucy Vallauri
}

\title{
Mimet (Bouches-du-Rhône) : à la recherche d'un atelier perdu
}

\author{
In: Archéologie du Midi médiéval. Tome 10, 1992. pp. 229-239.
}

\section{Résumé}

Dans le cadre des recherches sur la localisation des ateliers de potiers en Provence, la redécouverte d'un texte d'un érudit du début du XXe siècle signalant une officine à Mimet, dans l'arrière-pays marseillais, nous a amené à entreprendre une enquête dans les bibliothèques, les musées et sur le terrain afin d'en retrouver la trace. L'emplacement exact et la nature des productions ont pu être précisés. Les analyses géochimiques ont confirmé la cohésion de l'ensemble. Ce petit artisanat de céramique commune à pâte grise a sans doute alimenté la région de Marseille et d'Aix- en-Provence au tournant des XIP-XIIP siècles, avant d'être concurrencé par le centre varois d'Ollières et ceux plus importants de l'Uzège.

\section{Abstract}

As far as studies in the localization of ceramic work-shops in Provence are concerned, the rediscovery of a text written by a schola rat the beginning of the twentieth centwy, mentioning a factory at Mimet in the Marseille hinterland, led us to undertake both research in libraries and museums, and fleldwork in the area, in order to pick up the trail of the work-shop.

Its exact localization and the nature of ceramics produced have been identified. The geochemical data analysis corroborated the homogeneity of the entire production of vessels.

The potters of grey coarse ware supplied the areas of Marseille and Aix-en-Provence at the end of the twelfth century and the beginning of the thirteenth century, before being rivalled by the craftsmen of the Ollières centre in the Varand especially by the potters of Uzège.

Citer ce document / Cite this document :

Pelletier Jean-Pierre, Vallauri Lucy. Mimet (Bouches-du-Rhône) : à la recherche d'un atelier perdu. In: Archéologie du Midi médiéval. Tome 10, 1992. pp. 229-239.

doi : 10.3406/amime.1992.1231

http://www.persee.fr/web/revues/home/prescript/article/amime_0758-7708_1992_num_10_1_1231 


\title{
MIMET (Bouches-du-Rhône) : à la recherche d'un atelier perdu
}

\author{
Jean-Pierre PELLETIER et LUCY VALLAURI
}

Dans le cadre des recherches sur la localisation des ateliers de potiers en Provence, la redécouverte d'un texte d'un érudit du début du XX' siècle signalant une officine à Mimet, dans l'arrière-pays marseillais, nous a amené à entreprendre une enquête dans les bibliothèques, les musées et sur le terrain afin d'en retrouver la trace. L'emplacement exact et la nature des productions ont pu être précisés. Les analyses géochimiques ont confirmé la cohésion de l'ensemble. Ce petit artisanat de céramique commune à pâte grise a sans doute alimenté la région de Marseille et d'Aixen-Provence au tournant des XII'-XIII' siècles, avant d'être concurrencé par le centre varois d'Ollières et ceux plus importants de l'Uzège.

As far as studies in the localization of ceramic work-shops in Provence are concerned, the rediscovery of a text written by a scholar at the beginning of the twentieth century, mentioning a factory at Mimet in the Marseille hinterland, led us to undertake both research in libraries and museums, and fieldwork in the area, in order to pick up the trail of the work-shop.

Its exact localization and the nature of ceramics produced have been identified. The geochemical data analysis corroborated the homogeneity of the entire production of vessels.

The potters of grey coarse ware supplied the areas of Marseille and Aix-en-Provence at the end of the twelfth century and the beginning of the thirteenth century, before being rivalled by the craftsmen of the Ollières centre in the Var and especially by the potters of Uzège.

Dans le cadre des recherches entreprises au L.A.M.M. et au sein de l'association C.A.T.H.M.A. (1) sur les céramiques provençales cuites en atmosphère réductrice, ou plutôt selon le mode $B$ défini par M. Picon (Picon 1973), nous avons été amenés à nous intéresser à la seule mention connue pour la région marseillaise d'un four et d'un atelier de potiers médiévaux, mention figurant dans les revues anciennes et dont les passages principaux sont reproduits ci-dessous.

\section{1 - LES SOURCES ECRITES}

Un premier texte, extrait d'une monographie de Mimet (Gérin-Ricard 1903, p. 105-106), a été repris par son auteur de façon à peu près identique quelques années plus tard dans un bulletin consacré aux antiquités de la vallé de l'Arc (Gérin-Ricard 1907, p. 247-248):

"... Près du pont placé au sommet du fer à cheval décrit par la route qui va de Mimet à Simiane, nous avons découvert, il y a quelques années, les restes d'un atelier de potier (restes de murs, lits de cendres et de bois carbonisé très abondants, tessons appartenant à des vases qui n'ont jamais servi et ont été brisés, par la cuisson ou la destruction du hangar où ils étaient entreposés). Cet atelier placé près d'un ruisseau ou d'un banc d'argile qui apparaît aux écoles, occupe 15 mètres de façade sur la partie en déblais du chemin.

Cet atelier ne fabriquait que des vases minces et de forme élégante, quelquefois roses, mais la plupart offrent une teinte assez foncée dans la gamme des gris. On n'y trouve ni briques à rebords, ni dolia, ni amphores.

Les tessons que nous possédons se rattachent à sept formes différentes, avoir: type olla, avec anses plates, types à parois droites, type dit à tulype, deux types globuliformes à goulot étroit avec ornementation circulaire composée de stries verticales courtes ou de lignes pointillées peut-être à la roulette... Nous avons aussi à noter un type assez particulier d'urne cruciforme à col étroit et dont, la partie renflée est à parois plates formant des angles avec étranglement du col et celui du pied. De tous ces vases, un seul était apode et seul le type olla est pourvu d'anses.

La hauteur de ces vases varie entre 0,12 et 0,30 , leur diamètre à la panse va de 0,08 à 0,25 : leur ouverture est de 0,08 à 0,10 . Epaisseur de 0,002 à 0,005 .

La forme élégante de ces modèles, mais surtout leur ornementation formée de hachures, de points et d'un bourelet plat offrant des trous circulaires, rappellent certains vases trouvés dans les stations du lac Morat. Aussi l'origine de cette fabrique pourrait-elle être préromaine, mais comme aucune monnaie, aucun objet ne nous permet de lui assigner une date, même approximative, nous en sommes réduits aux hypothèses et à conclure seulement que cette figlina nous paraît d'assez haute époque : ses produits, fabriqués au tour, présentent des caractères assez particuliers et que nous avons rarement rencontrés dans la région.

En résumé, on fabriquait dans la vallée de l'Arc de la poterie grossière (tegulae, imbricae, dolia, vases moyens); l'atelier de Mimet est le seul que nous connaissons où l'on confectionnait l'article demi-fin. Nous employons cette expression parce que les spécimens de vases en question sont encore loin d'approcher de la finesse et de l'élégance des poteries importées par Marseille et qu'on rencontre dans les oppida et dans certaines villa du temps d'Auguste..."

Le Comte Henry de Gérin-Ricard était «Correspondant du Ministère de l'Instruction Publique, Secrétaire perpétuel de la Société de Statistique, Membre des Académies d'Aix et de Vaucluse, Membre de la Société Nationale des Antiquaires de France ". Son domaine de Valdonne se trouvait à quelques kilomètres de Mimet, et constituait le départ de ses " Excursions et recherches archéologiques en Provence", comme en témoigne ce titre d'un de ses articles (Gérin-Ricard 1931). Ses vastes connaissances alliées à l'intérêt qu'il portait aux découvertes fortuites effectuées dans la région ont fourni des témoignages irremplaçables. Parmi d'autres exemples,

(1) Laboratoire d'Archéologie Médiévale Méditerranéenne, et association Céramiques de l'Antiquité Tardive et du Haut Moyen Age, Université de Provence, 29 av. R. Schuman, 13621 Aix-en-Provence. 
on peut citer aussi le petit article «Plats d'argent contremarqués à l'époque mérovingienne trouvé à Valdonne..." (Gérin-Ricard 1901, p. 27-31): cette trouvaille a été exploitée récemment dans les Mélanges offerts à J. Lafaurie (Werner 1980, p. 257-263).

En 1942, dans un opuscule intitulé «La poterie carolingienne en Provence"), un autre érudit (Cabrens 1942) évoquait le même matériel de Mimet - matériel qu'il avait dû examiner - et présentait quelques croquis (Fig. 1), sans toutefois citer l'inventeur ni les publications antérieures:

"...Dans le midi de la France, la céramique gallo-romaine de l'époque mérovingienne avait des formes multiples: plats de diverses dimensions, coupes, écuelles à rebords ou munies d'oreilles, bols. A l'époque carolingienne..., en Provence, on ne connaît guère que trois formes, basses et ventrues : le pot à large orifice, le cruchon et le pégau. Ces vases sont d'un aspect gauche et d'une exécution rude. La pâte est très cuite, d'une couleur gris sale tirant souvent sur le noir, parfois gris bleuté.
Le pot à large orifice a généralement un rebord incurvé ; il est dépourvu de bec et possède une seule anse, courte et cannelée, partant du rebord $\left(\mathrm{n}^{\circ} 1\right)$. On trouve aussi un type à parois droites et épaisses et un autre, sans anses, ayant la forme du jarron actuel. Hauteur: environ trente centimètres.

Le cruchon est l'analogue de notre gargoulette $\left(n^{\circ} 2\right)$. Nous n'avons pu déterminer s'il avait un couvercle.

Le pégau ou pichet est l'ancêtre de notre cruche. C'est un vase à fond plat, sans col, et à panse à peu près aussi large que haute (dix à quinze centimètres). De l'orifice part une anse en arc de cercle; un bec se trouve à l'opposé $\left(\mathrm{n}^{\circ} 3\right)$. Dans certains vases l'ourlet de l'orifice n'est pas interrompu par le bec et forme aussi un pont pouvant servir de deuxième anse (ce type ne se trouve qu'exceptionnellement en Provence, ainsi que les pégaus à bec énorme qui semblent cantonnés dans le Languedoc). Le pégau provençal a un couvercle qui porte en dessous deux excroissances triangulaires destinées à le maintenir quand on incline le vase...

Mimet. - Dans les Bouches-du-Rhône, au pied du village de Mimet et près d'une chapelle méro-carolingienne, N.-
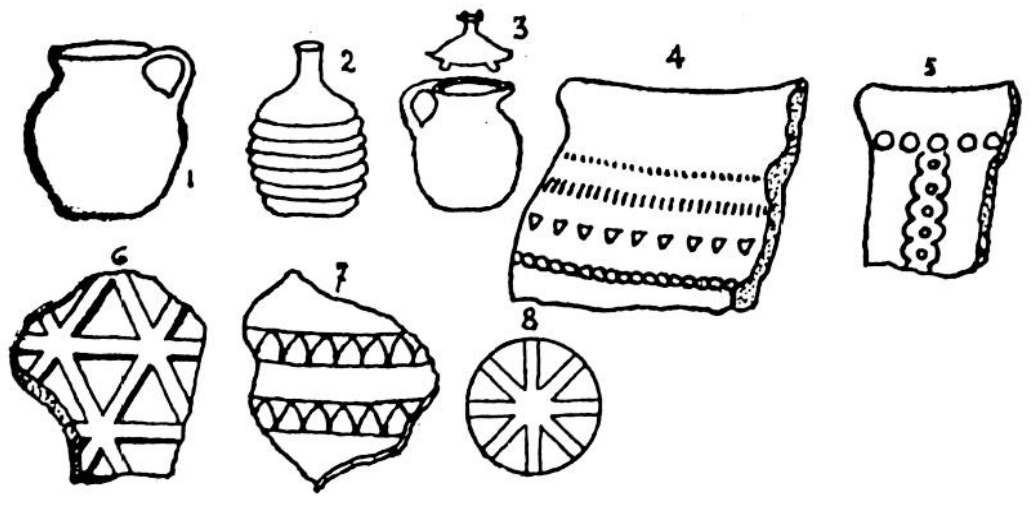

Fig. 1: Formes et décors évoqués par R. de Cabrens (1942, p. 6).

D. du Cyprès, on a découvert, il y a quarante ans environ, un four et un atelier de potiers.

Les rebuts de fabrication, assez abondants, nous ont livré des débris de vases se rapportant aux trois formes décrites cidessus. Quant au décor, nous avons relevé les types suivants :

$1^{\circ}$ Stries incisées à la roulette et diposées en bandes faisant le tour du vase. Elles se présentent le plus souvent sous forme de traits verticaux. Ces traits sont parfois réduits à la dimension de points ou offrent, plus rarement, l'aspect de triangles ou d'une bande cordée. Nous avons représenté ces divers types sur la même figure $\left(n^{\circ} 4\right)$.

$2^{\circ}$ Impressions digitales: elles ne se rencontrent que sur les marmites ou jarrons à parois épaisses et ornent la partie supérieure du vase dont elles font le tour. Des bandes de pâte rapportées et appliquées sur le vase dans le sens vertical sont ornées aussi d'impressions circulaires, mais plus petites $\left(n^{0} 5\right)$. Ces impressions sont parfois carrées...

$3^{\circ}$ Côtelures : beaucoup de vases présentent extérieurement des lignes circulaires faites au tour, constituant ainsi un décor annelé. Parfois ces simples traits deviennent de véritables côtelures $\left(\mathrm{n}^{\circ} 2\right)$.

$4^{\circ}$ Décor géométrique: bandes de triangles estampés offrant un décor en croix de Saint-André $\left(n^{\circ} 6\right)$.

Bandes de chevrons incisés $\left(n^{\circ} 7\right)$.

$5^{\circ}$ Rouelles estampées: la rouelle, comme nous l'avons déjà dit, est caractéristique du décor mérovingien. Motif ornemental venu d'Orient avec l'influence byzantine du IV ${ }^{\mathrm{C}}$ siècle elle se présentait sous des formes diverses : cercle pointillé ou double renfermant un petit cercle, roue ou rosace, palme circulaire, soleil ou soleil tournant, cercles concentriques. Elle était estampée légèrement dans une pâte très fine de couleur gris clair, plus rarement rouge orangé; et était associée en général à des palmes, parfois à un autre motif.
La rouelle carolingienne $\left(\mathrm{n}^{\circ} 8\right)$, ou que nous croyons telle, est très différente. Profondément estampée dans une pâte grossière, elle se compose de rayons rigides assez nombreux. C'est, du reste, un décor assez rare. Nous n'en avons trouvé qu'à Mimet et à Saint-Blaise (St-Mitre)...»

\section{2 - LE SITE ET L'ENQUÊTE SUR PLACE (2)}

Sur un piton accroché au flanc nord de la grande barrière naturelle que forme la chaîne de l'Etoile entre le bassin d'Aix et celui de Marseille, le petit village de Mimet dont le nom apparaît au $X^{\mathrm{I}}$ siècle est le plus élevé des Bouches-du-Rhône, à cinq cents mètres d'altitude (Fig. 2). Depuis le château féodal, la vue est pratiquement illimitée à l'ouest et au nord. En contrebas de l'agglomération, le site décrit par Gérin-Ricard a été profondément transformé par les réfections de la route départementale $n^{\circ} 8$, l'aménagement du virage «en fer à cheval " (Fig. 3), d'un terrain de sport, et de nombreuses constructions individuelles. Il ne subsiste plus aucune trace des installations décrites au début du siècle, ni aucun souvenir dans la mémoire des habitants du village.

L'enquête sur le terrain, auprès des services municipaux et dans les archives permit sinon de localiser le site avec précision, du moins de retrouver la trace de Guy Balansard, pharmacien à Marseille et propriétaire du domaine voisin de La Verrerie. En 1965, il avait été intrigué par une fosse mise en évidence par un bulldozer qui élargissait un chemin dans la pente, à une dizaine de mètres au-dessus du virage de la route départementale.

(2) Nous tenons à remercier toutes les personnes qui ont facilité cette enquête : G. Balansard; Mesdames Blanc; G. Bertucchi, Conservateur des Antiquités; la Municipalité de Mimet; Y. Guadagni, président de l'association des A Amis de l'Óst ; A. Tarin et A. Durand, Conservatrices des musées de Marseille; et Y. et J. Rigoir pour leur fidèle collaboration. 


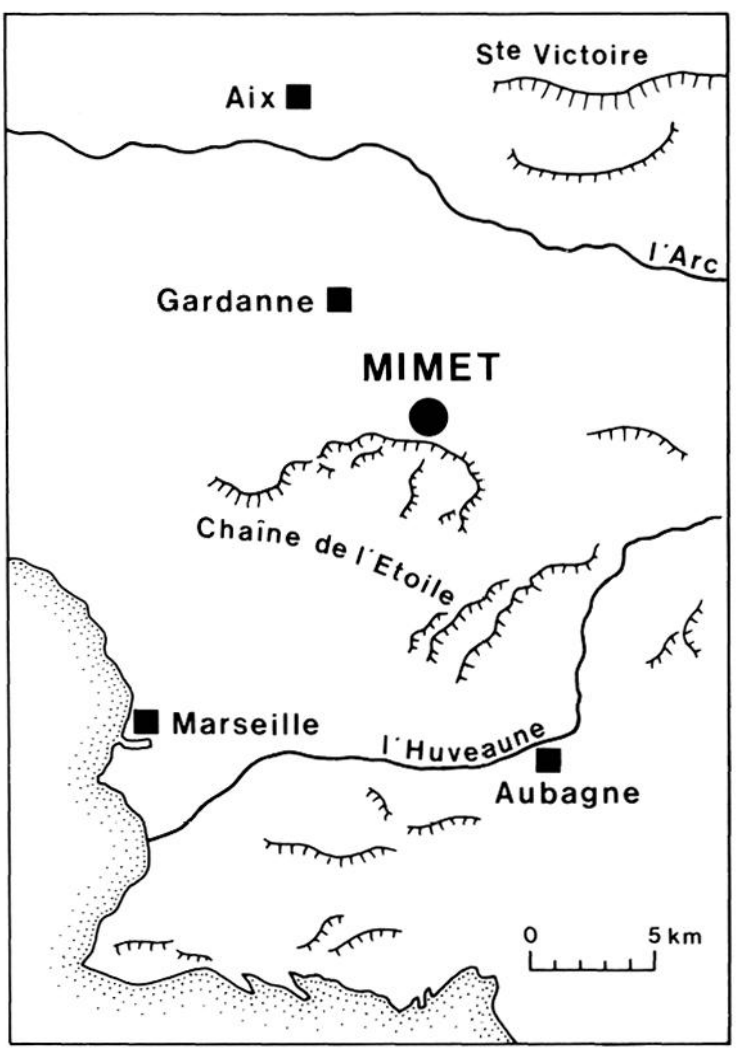

Fig. 2: Situation de Mimet dans l'arrière-pays marseillais.

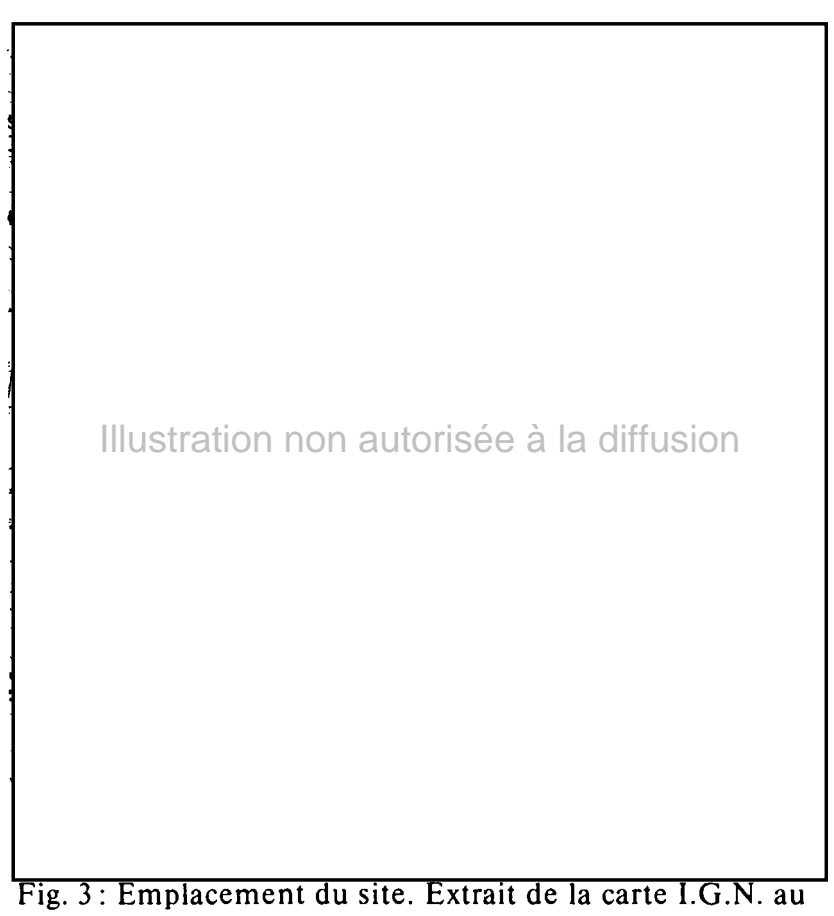
$1 / 20000^{\mathrm{c}}$ de 1936 .

Cette fosse, creusée dans la terre naturelle, était longue de $1,5 \mathrm{~m}$, large de $1 \mathrm{~m}$, profonde de $1 \mathrm{~m}$ et de forme ovoïde. G. Balansard, constatant que la structure avait été "énormément abîmée", fouilla ce qui en restait afin d'éviter un pillage. L'argile rouge granuleuse constituant le comblement apparaissait bien différente des argiles naturelles locales. Il ne s'y trouvait aucun ossement, mais quelques tessons de poterie grise parfois décorés à la roulette, ainsi qu'une monnaie de Guillaume de Forcalquier, datable de 1209-1220. Il en informa la Direction des Antiquités de l'époque par un courrier que nous avons retrouvé au S.R.A. d'Aix-enProvence, bien classé dans le dossier consacré à Mimet, avec deux petits échantillons de céramique. L'affaire en resta là.

Une quinzaine d'années plus tard, les terrains de ce lieu-dit «La Pignatelle» furent morcelés. En provençal, pignato signifie marmite ou pot de terre; si les toponymes issus de pignate sont très répandus en Provence orientale, La Pignatelle à Mimet constitue le seul exemple connu dans les Bouches-du-Rhône d'après le fichier Rivoli établi à partir du cadastre actuel (renseignement $\mathrm{H}$. Amouric).

En bordure de la parcelle acquise par Mesdames Blanc, sur laquelle G. Balansard avait observé la fosse, un nouveau chemin fut creusé dans la pente pour accéder aux autres propriétés. Autour de leur pavillon, dans les terres remuées par l'engin et en plantant leurs massifs de rosiers, Mesdames Blanc recueillirent un certain nombre de tessons de poterie grise et des fragments de tuiles canal de fabrication très fruste.

En 1988, nos prospections systématiques sur place permirent de récolter encore des tessons, ainsi que dans les coupes en amont de ce nouveau chemin, sur quelques dizaines de mètres. On avait donc de sérieux indices de la présence dans cette zone de l'atelier décrit par Gérin-Ricard.

Notre collègue $J$. Rigoir nous indiqua que les collections de Gérin-Ricard avaient autrefois été déposées en partie au Musée Borély de Marseille. A l'occasion de la réorganisation des musées de Marseille, nous avons pu avoir accès aux réserves qui n'avaient pas encore été déménagées. Aucun tesson de Mimet ne semblait s'y trouver. C'est par un pur hasard que dans l'atelier des ouvriers, dans un meuble à plans difficilement accessible derrière un tas de planches, nous avons retrouvé des fragments de vases étiquetés "Mimet B.d R. » (Fig. 4); dans ce même tiroir se trouvait une carte d'adhérent du Comité Socialiste Indépendant de 1930, au dos de laquelle, à la plume, était noté "Tranchée de la route près Mimet Vasseur 1910" (Fig. 10). Il s'agit sans aucun doute de l'archéologue du début du siècle, passionné entre autres par les céramiques régionales et qui avait notamment écrit un article sur la céramique estampée (Vasseur 1907), en fait la DS.P. chère à Y. et J. Rigoir.

\section{3 - LE MATERIEL}

Les 28 tessons recueillis par G. Balansard, ajoutés aux 88 que Mesdames Blanc et nous-mêmes avons récolté sur place (dont un qui a recollé avec ceux de la cruche de $1965 \mathrm{n}^{\circ} 5$ fig. 7), ainsi que les quinze gros fragments du Musée Borély constituent un ensemble homogène. Malgré les incertitudes découlant des conditions de découverte, les dimensions relativement importantes d'un certain nombre de tessons permettent d'esquisser, ou plutôt de donner quelques éléments de la typologie des productions de l'atelier.

Tous ces échantillons ont une pâte fine, peu granuleuse, contenant des petites inclusions blanches, et sont de teinte gris à gris clair le plus souvent, parfois beige rosé, comme l'indiquait Gérin-Ricard. Ces variations de couleur résultent d'une réduction incomplète lors de la post-cuisson.

\section{1. - Les formes}

\subsection{1. - Les pégaus, ou pots (Fig. 5)}

Sans toutefois se hasarder à interpréter des comptages, les pégaus apparaissent comme étant ici le type d'objet le plus représenté. Ces petits pots sont bien connus en Provence, dans les sites d'habitat et les nécropoles (Démians d'Archimbaud 1980, p. 292-297). 

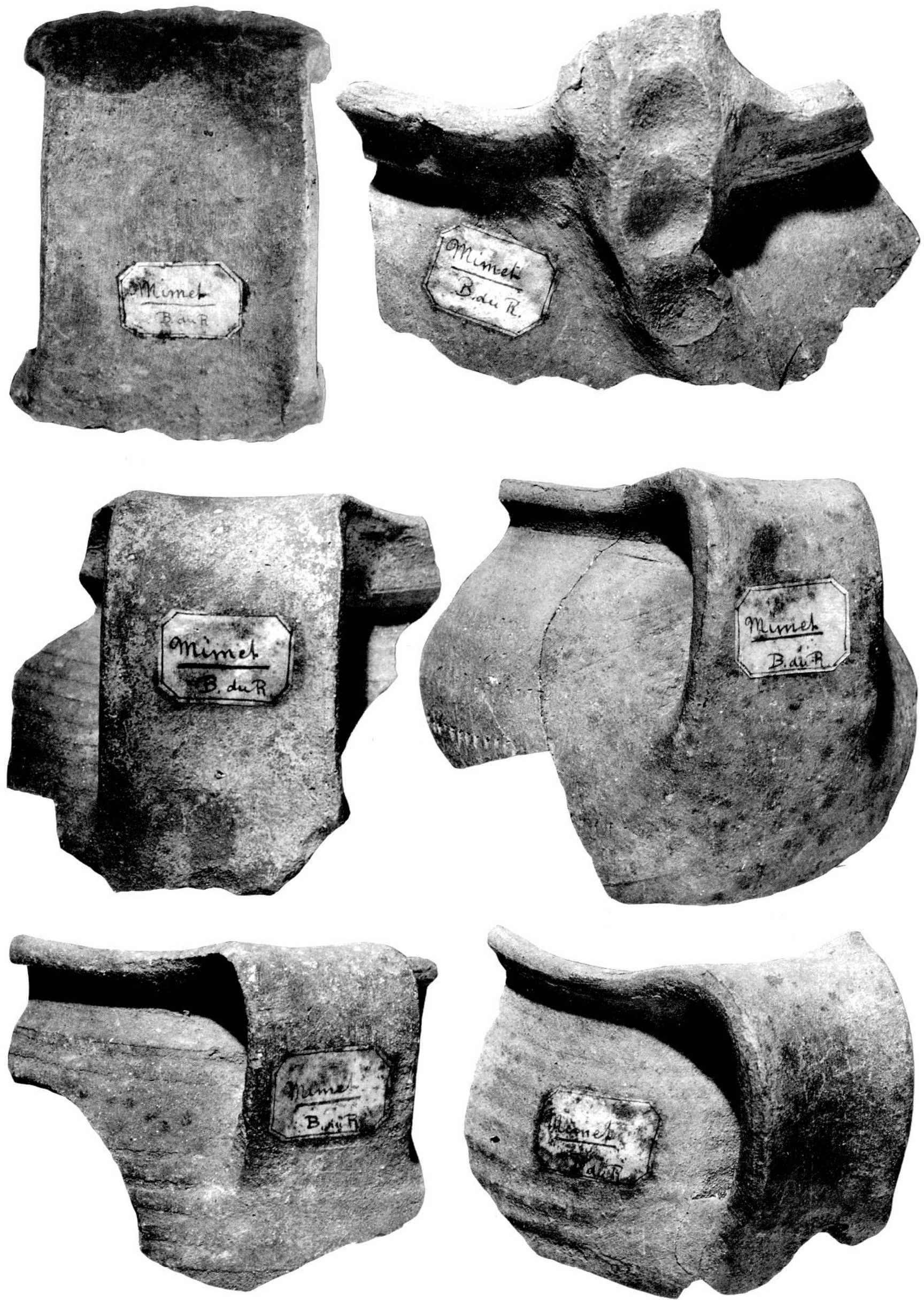

Fig. 4 : Quelques-uns des fragments découverts au Musée Borély $(\mathrm{Cl}$. Y. Rigoir). Réd. $90 \%$. 


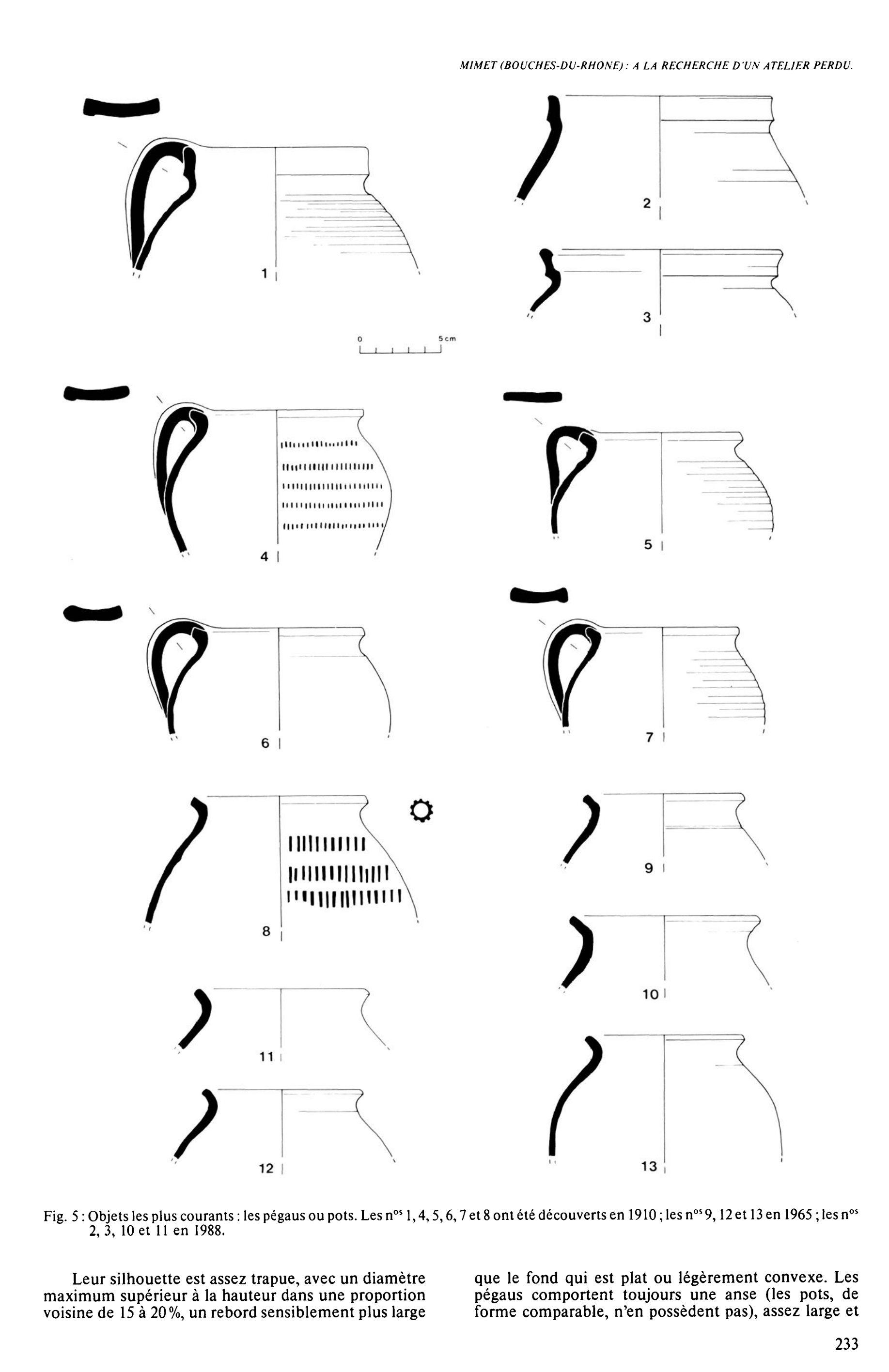


peu épaisse, et rarement un bec pincé. Ils n'ont pas de col: la panse bien arrondie commence immédiatement sous le rebord.

Les exemplaires de Mimet peuvent être classés en trois catégories:

- avec rebord en bandeau ou en poulie (Fig. $5 n^{\circ} 1$ à 3), - avec rebord bien structuré, plus épais que la panse (Fig. $5, n^{\circ} 4$ à 8 ). On retrouve ce typc de rebord sur un gros pot (Fig. $6, n^{\circ} 2$ ), et sur un pégau (contexte 427 , four S. 103) découverts à Marseille Sainte-Barbe.

- avec rebord simple, de même épaisseur que la panse (Fig. $5, \mathrm{n}^{\circ} 9$ à 13).

Les pégaus ou pots à rebord en poulie apparaissent sensiblement plus grands (diamètre au rebord 12 à $14 \mathrm{ou}$ $15 \mathrm{~cm}$, mais le fragment $\mathrm{n}^{\circ} 3$ est peut-être déformé) que les autres (en moyenne 10 à $11 \mathrm{~cm}$ pour le diamètre du rebord, 13 à $15 \mathrm{~cm}$ pour le diamètre maximum). Les attaches d'anses sont exclusivement du type «incluant le rebord", et les anses sont à section simple, rubanée. Quelques panses sont animées de raies de tournage. Deux objets sont décorés à la roulette; pour le $n^{\circ} 8$, le diamètre de l'instrument, $14 \mathrm{~mm}$, donne un motif long de $44 \mathrm{~mm}$ comportant au $\mathrm{cm}$ trois dents larges de 9 à $10 \mathrm{~mm}$.

\subsection{2. - Les grands pots (Fig. 6)}

Cinq fragments appartiennent à des pots dont les dimensions sont nettement plus importantes que celles des pégaus. Ce sont des objets d'usage peu courant, encore mal connus dans notre région (Démians d'Archimbaud 1980, p. 304). Une de leurs caractéristiques est l'attache supérieure des anses, appliquées sous le rebord (Fig. $6, n^{\circ} 1,3$ et 4 ) ou sur la panse ( ${ }^{\circ} 6$ et 7 ). Ces anses, à la section rubanée ou légèrement cannelée, peuvent être très larges.

L'objet $\mathrm{n}^{\circ} 4$ comporte un rebord dont le profil évoque celle de certains pégaus (Fig. $5, \mathrm{n}^{\circ} 4$ à 7 ). Il présentc en outre une ressemblance évidente avec un gros fragment (Fig. $6, n^{\circ} 2$ ) découvert tout récemment dans un contexte du XIII' siècle à Marseille Sainte-Barbe (Marchesi 1992), mais dont la composition géochimique est différente.

Le rapprochement s'impose aussi avec une forme complète (Fig. $6, n^{\circ} 5$ ) provenant du comblement dans la deuxième moitié du XIII' siècle d'un silo du palais archiépiscopal d'Aix-en-Provence (Fixot 1985, p. 47, fig. 75 et Fixot 1989, p. 57, fig. 31). Cette pièce au rebord de même type comporte deux anses; la présence d'un bec tubulaire placé au sommet de la panse, sous la lèvre, perpendiculairement aux anses, indique clairement la fonction de stockage pour les liquides.

\subsection{3. - Eléments de formes diverses (Fig. 7)}

Les rebords $n^{\circ} 1,2$ et 3 ne semblent pas appartenir aux catégories précédentes.

Les cruches, avec un col bien marqué et un bec pincé ( $\mathrm{n}^{\circ} 4$ et 5 ), sont une forme assez rare dans le répertoire des céramiques grises en Basse-Provence (Démians d'Archimbaud 1980, p. 302-303). La roulette utilisée pour le décor de la panse de l'objet $n^{\circ} 5$, avec un diamètre de $19 \mathrm{~mm}$, donne un motif de $60 \mathrm{~mm}$ comportant au $\mathrm{cm}$ trois à quatre dents larges de $6 \mathrm{~mm}$.

Le fragment d'une anse verticale $\left(n^{\circ} 6\right)$, malgré un bourrelet latéral marqué, reste de section simple.

Le rebord $\mathrm{n}^{\circ} 7$ appartient à une jatte ou une écuelle: les formes ouvertes sont rares elles aussi (Démians d'Archimbaud 1980, p. 303-304).

Les fonds, plats $\left(n^{\circ} 8-9\right)$ ou légèrement bombés $\left(\mathrm{n}^{\circ} 10\right.$ à 13$)$ ont un diamètre qui semble un peu trop important pour les attribuer à des pégaus de dimension moyenne. L'angle compris entre la base de la panse et l'horizontale est très variable, entre $45^{\circ}$ et $70^{\circ}$. Le fond $\mathrm{n}^{\circ} 14$, en pâte beige, provient d'un très grand vase; le $n^{\circ} 15$ sans doute d'un très petit pégau.

\subsection{4. - Les formes rares (Fig. 8)}

Ces fragments apparaissent assez exceptionnels. Le rebord à section en poulie du très grand vase $n^{\circ} 1 \mathrm{com}$ porte une préhension en forme d'anse de panier au décor digité.

Le $\mathrm{n}^{\circ} 2$, très épais, avec un diamètre de $36 \mathrm{~cm}$, doit provenir d'un très grand récipient destiné au stockage. Il est renforcé, au sommet de la panse, par un cordon horizontal.

Le fragment de panse $n^{\circ} 4$ implique un vase aux parois presque cylindriques, très épaisses $(15 \mathrm{~mm})$, pour un diamètre de $18 \mathrm{~cm}$ seulement, et comportant six bandes verticales rapportées de cordons digités.

L'anse ou poignée circulaire $\mathrm{n}^{\circ} 5$, de grande cruche ou gourde, avec sa section en $U$, est tout à fait comparable à un fragment découvert à Rougiers dans un niveau daté de la fin XII'-première moitić du XIII ${ }^{\circ}$ siècle (Démians d'Archimbaud 1980, p. 304 et fig. 261, $\left.n^{\circ} 6\right)$.

Le couvercle à ergots $n^{\circ} 3$ ressemble - ou correspond? - à celui dessiné par R. de Cabrens (Fig. 1, $\mathrm{n}^{\circ} 3$ ). Les seuls autres objets connus de ce type ont été découverts dans un contexte de l'an Mil (Fixot 1983, p. 101 et fig. $\left.10, n^{\circ} 7-8\right)$. Les couvercles à ergots ne peuvent s'adapter que sur certaines formes de rebords dont nous n'avons pas d'exemple ici, sauf peut-être le pot $n^{\circ} 4$, Fig. 6?

Avec les éléments dont nous disposons, les sept formes différentes évoquées par Gérin-Ricard restent difficiles à préciser.

\section{2. - Comparaisons, datations et observations.}

Ce qui frappe de prime abord à l'examen de ce matériel, c'est l'absence quasi totale de fragment - rebord, anse, panse ou fond - attribuable aux marmites globulaires bien répandues en Provence dans la première moitié du XIII ${ }^{\circ}$ siècle, sinon dès la fin du XII ${ }^{*}$ siècle (Phase Al de Rougiers, Démians d'Archimbaud 1980 , p. 297-302). A ces marmites sont généralement associés des couvercles de forme applatie: aucun en semble attesté à Mimet, pas plus que le décor à la molette fréquent sur ces deux types d'objets (Démians d'Archimbaud 1980, p. 306 ; Fixot 1989, p. 57, fig. 31).

Il semble ainsi que nous soyons plus proches de l'héritage des formes de l'an Mil, lesquelles ont perduré sans doute jusque vers la fin du XII ${ }^{\circ}$ siècle, que des productions d'Ollières au début du XIII" siècle.

En ce qui concerne les pégaus, les départs d'anses incluant le rebord, type de fixation ancien, sont les seuls représentés.

Les descriptions de Gérin-Ricard de « vases minces et de forme élégante" semblent plutôt se rapporter à des pots ou des pégaus, mais pas aux tessons épais des grosses jarres de la Fig. 8, dont certains avec les décors de cordons peuvent avoir été vus par R. de Cabrens. M. Vasseur est-il revenu sur le site en 1910, quelques années après les premières découvertes, mettant au jour des matériels de nature parfois différente?

Les remarques de $R$. de Cabrens concernant les décors de rouelles estampées doivent être attribuées à la présence de tessons de DS.P. dans les lots qu'il a pu observer: des ramassages récents ont confirmé dans le quartier des traces de l'Antiquité tardive. Une villa gal- 

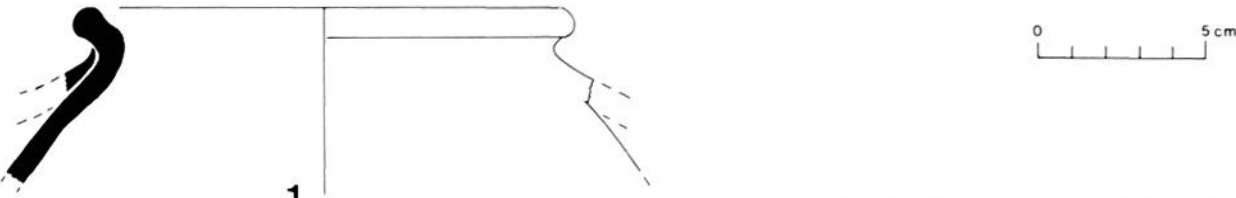

1
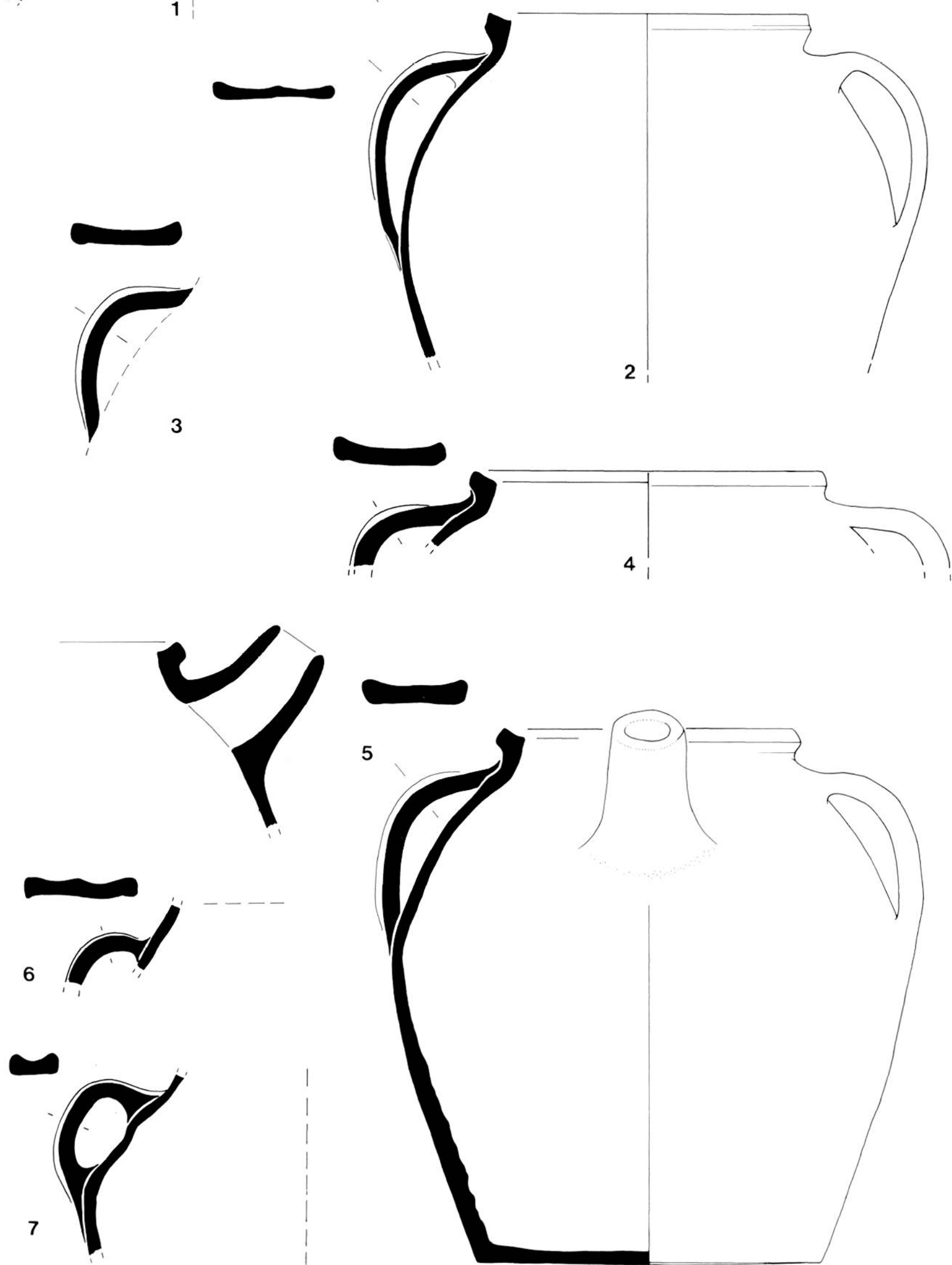

Fig. 6: Gros pots et jarres à anses verticales. $N^{\circ s} 1$ et $6:$ Mimet $1988 ; n^{05} 3,4$ et $7:$ Mimet $1910 ; n^{\circ} 2:$ Marseille Sainte-Barbe ; $n^{\circ} 5$ : Aix, palais de l'archevêché. 


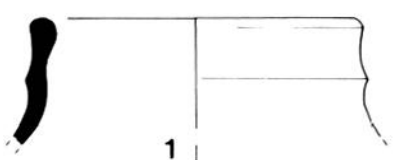

1
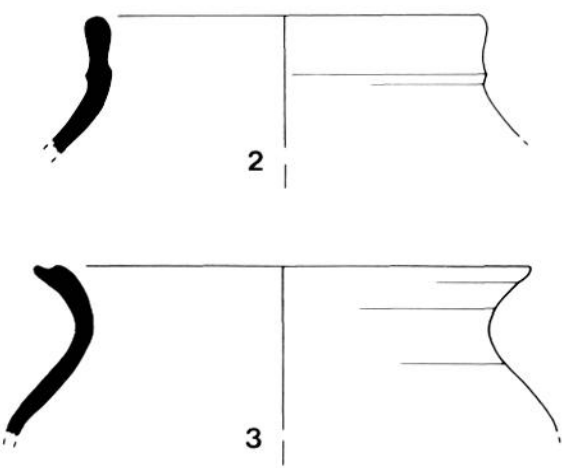

3
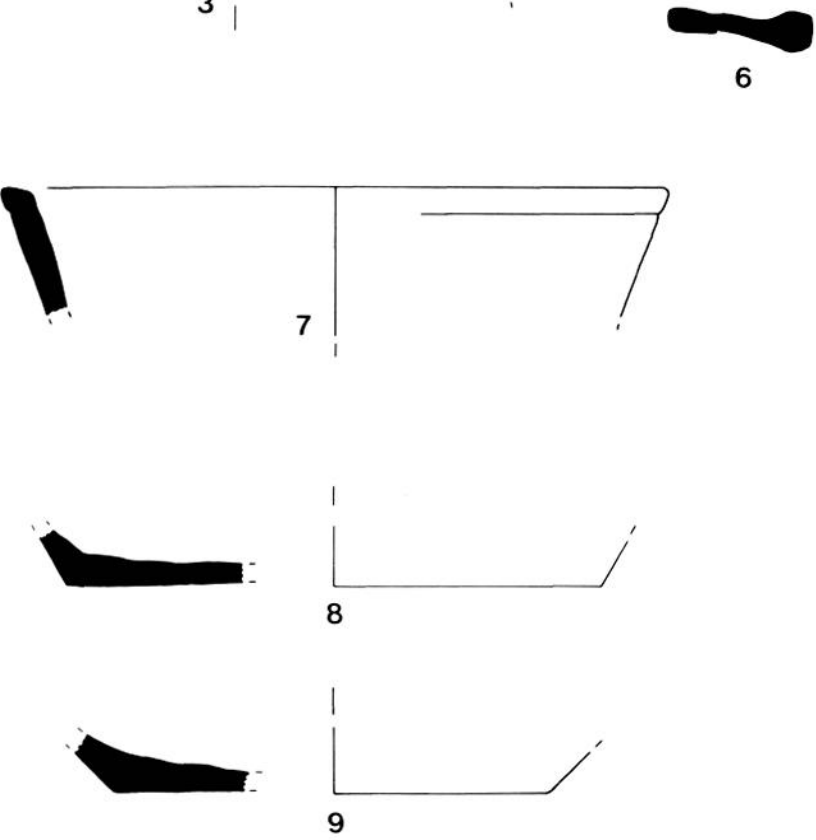

6
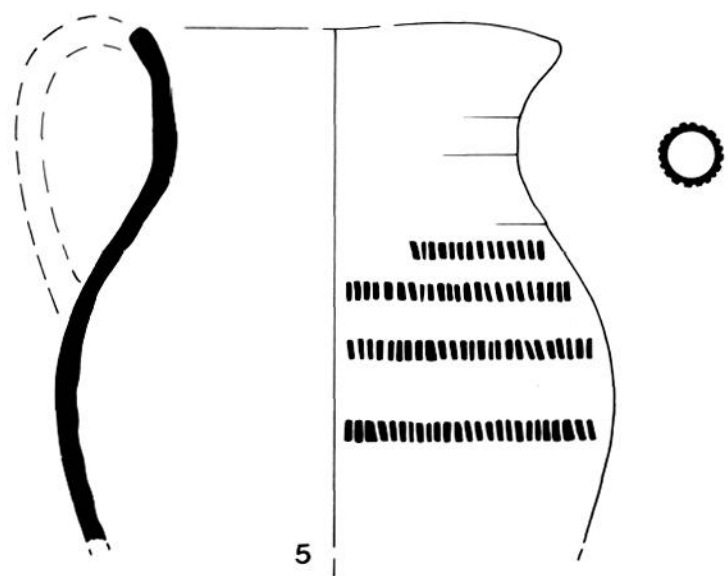

IIIIIIIIII

|IIIIIIIIIIIIIIIIIIIIII

IIIIInก)

InaIIIIIIIIIIIIIII)

5
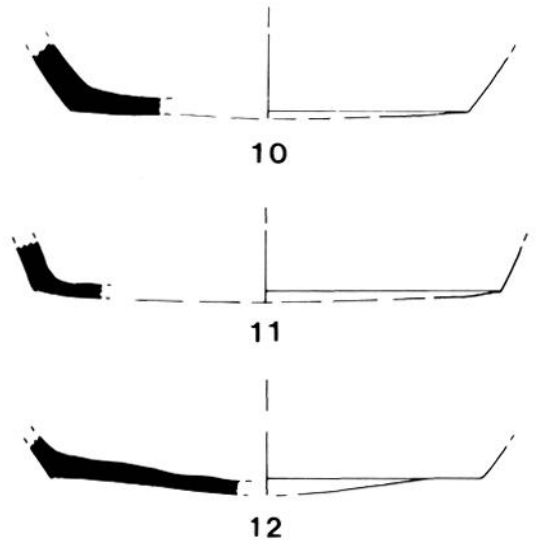

12

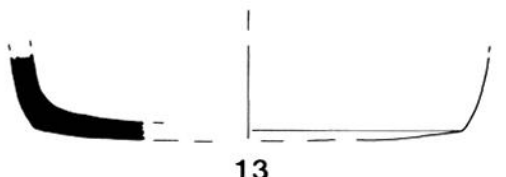

13
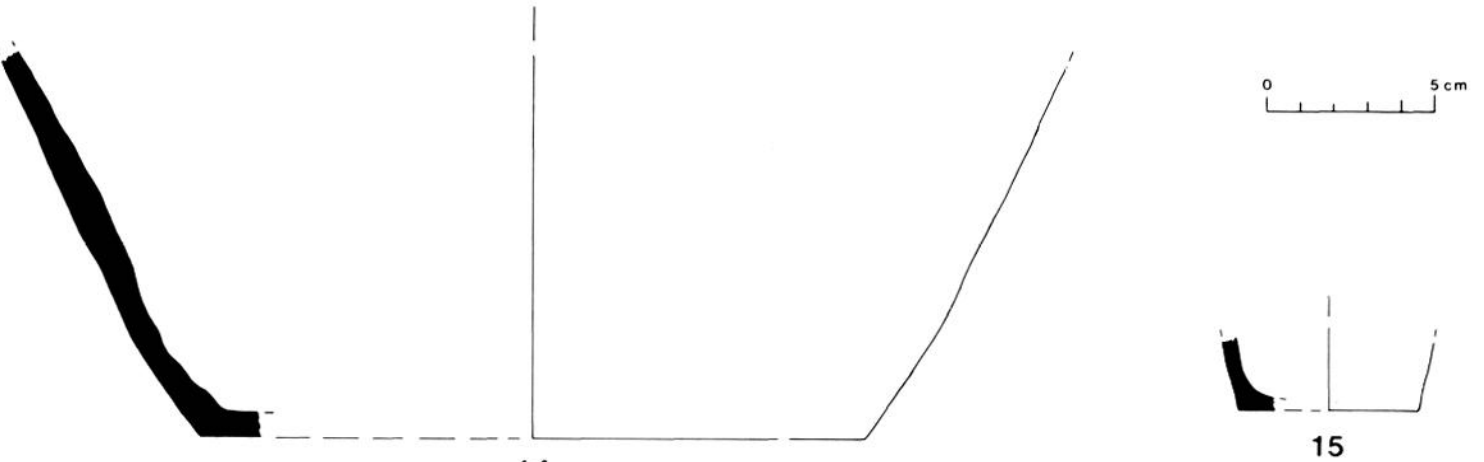

15

Fig. 7: Eléments de formes diverses. $\mathrm{N}^{\circ} 3$ et 5 : ramassage 1965 ; autres: ramassages 1988.

lo-romaine a été signalée par Gérin-Ricard à quelques minutes de marche (Gérin-Ricard 1903, p. 105). Quelques tessons de la période protohistorique ont aussi été retrouvés, qui ajoutent à la permanence de l'occupation des environs immédiats.

Donc l'ensemble des formes, souvent archaïsantes, présente des indices d'antériorité par rapport aux productions connues notamment à Ollières et à Pourcieux. Si la durée de fonctionnement de cet atelier ne saurait être définie avec précision, on peut néanmoins le situer, en fonction du contexte régional, au tournant du $\mathrm{XII}^{\mathrm{c}}$ au XIII' siècle.

\section{4 - LES ANALYSES EN LABORATOIRE}

Actuellement, une première série d'analyses réalisées par M. Picon (Laboratoire de Céramologie de Lyon), portant sur cing échantillons de couleur et de texture sensiblement différente à première vue, a con- 
firmé l'originalité de l'atelier de Mimet (Fig. 9). Sur ce dendrogramme, chaque trait vertical du bas représente une céramique analysée; les traits horizontaux reliant ces derniers sont d'autant plus bas que les compositions des céramiques se ressemblent.

Parmi ces échantillons figurent le bord $n^{\circ} 2$, fig. 7 (AMM 260), le fond $n^{\circ} 12$, fig. 7 (AMM 262) et le pégau $\mathrm{n}^{\circ} 4$, fig. 5 (AMM 264), ainsi qu'un fragment de panse de gros pot (AMM 263) et un rebord simple de pégau com- parable au n ${ }^{\circ}$, fig. 5 (AMM 261).

Les compositions chimiques sont assez différentes de celles de la plupart des autres céramiques grises étudiées, datant de l'Antiquité tardive. Les échantillons médiévaux constituent un ensemble très homogène qui s'intègre dans un groupe comprenant des découvertes effectuées dans les environs, à Gardanne (Pelletier 1991), Peypin et Simiane-Collongue (Pelletier à paraîtrc).
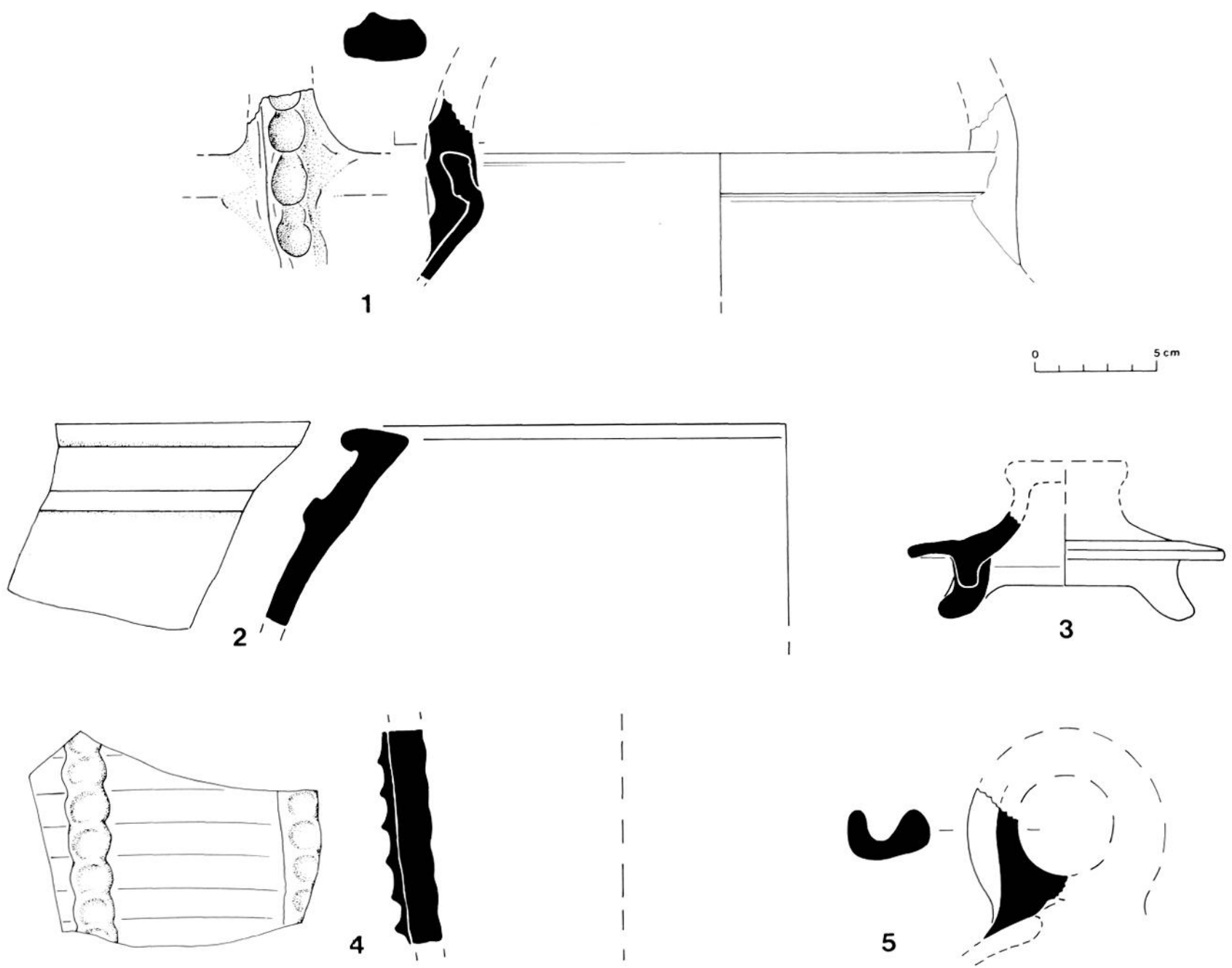

Fig. 8: Formes rares. Nº 1 à 4 : découvertes Vasseur $1910 ; n^{\circ} 5$ : ramassage 1988.

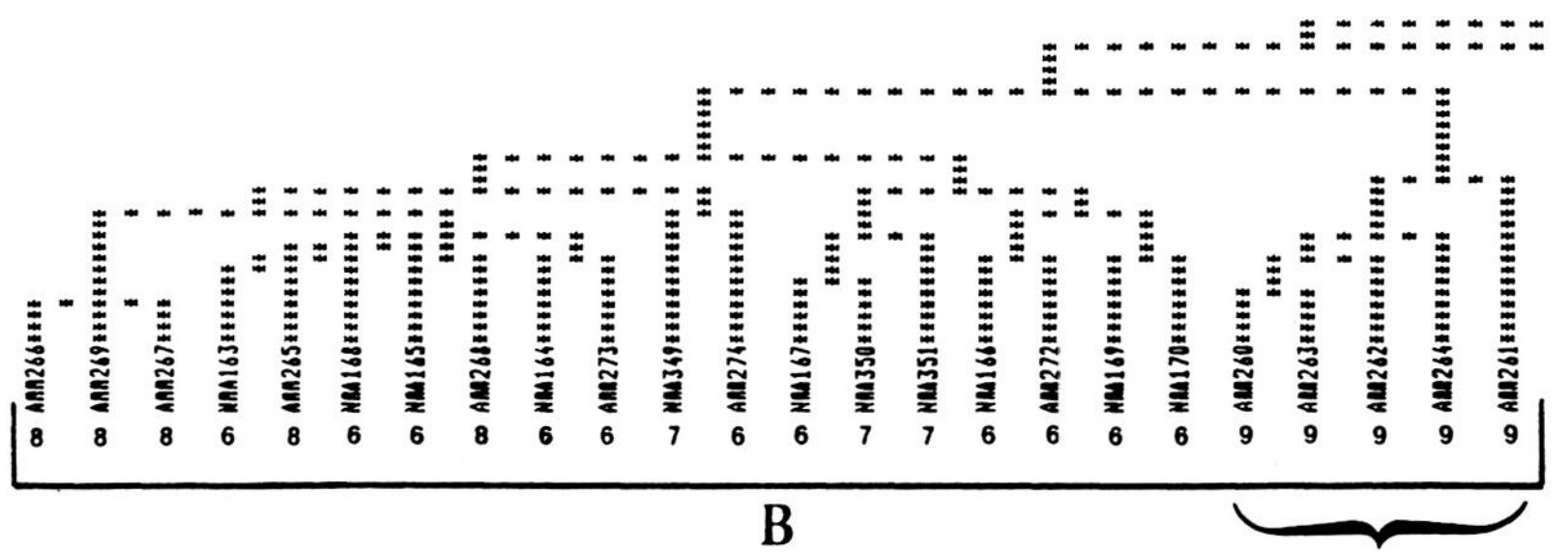

Fig. 9 : Extrait du dendrogramme publié dans les Actes du Colloque International de Rabat. Ce groupe B comprend exclusivement des communes grises de l'arrière-pays marseillais: Gardanne $\left(n^{\circ} 6\right)$, Peypin $\left(n^{\circ} 7\right)$, Simiane-Collongue $\left(n^{\circ} 8\right)$, trois sites de l'Antiquité tardive. Les échantillons de Mimet $\left(n^{\circ} 9\right)$ sont localisés dans la grappe à droite du groupe. 


\section{CONCLUSION}

Dans l'état actuel de cette enquête, si aucune des structures décrites autrefois n'a été retrouvée, la présence de cet atelier ne fait aucun doute. L'homogénéité des productions collectées, et leur typologie particulière sont confirmées par les analyses géochimiques.

La localisation de cette officine à proximité immédiate du village est des plus classiques au moyen âge et à l'époque moderne (Amouric 1986, p. 606). C'est le cas des ateliers médiévaux d'Ollières et de Saint-Gilles du Gard (Leenhardt 1989).
Ainsi, cet atelier aujourd'hui disparu avait perpétué une tradition régionale vieille de près de sept siècles, ou avait renoué avec elle. Les potiers de Mimet n'ont pu opérer les mutations technologiques qui s'imposèrent dans le courant du XIII ${ }^{\circ}$ siècle et contribuèrent au rayonnement d'Ollières, à $25 \mathrm{~km}$ de là. On peut évoquer aussi le problème d'une éventuelle concurrence.

La poursuite des recherches entreprises devrait permettre de préciser le répertoire de formes, l'importance de cette officine ainsi que la diffusion de ses produits vers les villes proches.
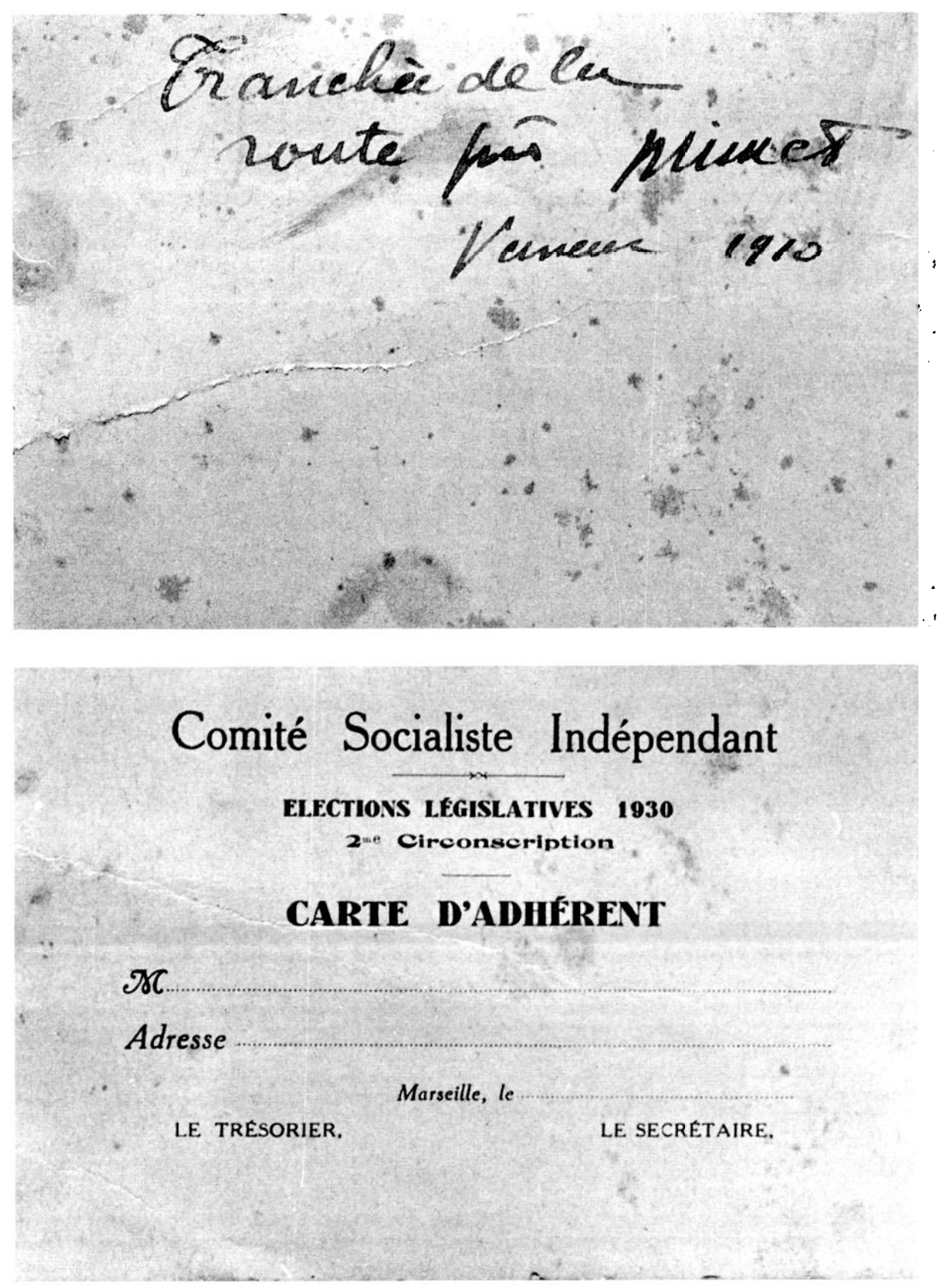

Fig. 10: Létiquette en papier rouge jointe aux fragments découverts au Musée Borély (Cl. Y. Rigoir). 


\section{BIBLIOGRAPHIE :}

Amouric 1986: AMOURIC (H.), DEMIANS D'ARCHIMBAUD (G.). - Potier de terre en ProvenceComtat Venaissin au Moyen Age, Artistes, Artisans et production artisitique au Moyen Age, volume I, les hommes 1986, p. 601-603.

Cabrens 1942 : CABRENS (R. de). - I a poterie carolingienne en Provence, Toulon, 1942, 15 p.

Demians d'Archimbaud 1980 : DEMIANS D'ARCHIMBAUD (G.) - Les fouilles de Rougiers, contribution à l'archéologie de l'habitat rural médiéval en pays méditerranéen, Paris, C.N.R.S., 1980.

Demians d'Archimbaud 1985 : DEMIANS D'ARCHIMBAUD (G.) - La production des céramiques médiévales en Provence centrale ; état des questions ; Histoire des techniques et sources documentaires, cahier $\mathrm{n}^{\circ} 7$, Actes du colloque du G.I.S. Aix-en-Provence, octobre 1982, Aix 1985, p. 141-156.

Fixot 1983: FIXOT (M.), PELLETIER (J.-P.), Une forme originale de fortification médiévale provençale : le castelas de Cucuron (Vaucluse), Archéologie Médiévale, XIII, 1983, p. 89-115.

Fixot 1983 : FIXOT (M.), GUYON (J.), PELLETIER(J.P.), RIVET (L.). - Des abords du forum au palais archiépiscopal, étude du centre monumental d'Aixen-Provence, Bulletin Monumental, t. 144-III, 1986, p. $195-290$.

Fixot 1989 : FIXOT (M.), GUYON (J.), PELLETIER(J.P.), RIVET (L.) - Le palais archiépiscopal d'Aix-enProvence, notice 19, p. 56-58, L'église et son environnement, Archéologie Médiévale en Provence, Catalogue de l'exposition du Musée Granet, Aix, 1989.

Gérin-Ricard 1901 : GERIN-RICARD (H. de). - Plats d'argent contremarqués à l'époque mérovingienne trouvés à Valdonne (Bouches-du-Rhône), Bulletin Archéologique du Comité des Travaux Historiques, 1901, p. 27-31.

Gérin-Ricard 1903 : GERIN-RICARD (H. de). - Mimet et Notre-Dame-des-Anges, Monographies de Communes, fasc. $\mathrm{n}^{\circ} 3$, Marseille, 1903, p. 103-109.

Gérin-Ricard 1907: GERIN-RICARD (H. de) et
ARNAUD D'AGNEL (G.). - Les Antiquités de la vallée de l'Arc en Provence, Publications de la Société d'Etudes Provençales, Aix, 1907, 334 p.

Gérin-Ricard 1901 : GERIN-RICARD (H. de). - Excursions et recherches archéologiques en Provence. Provincia, XI, 1931, p. 160-168.

Leenhardt 1989 : LEENHARDT (M.), THIRIOT (J.). Poteries grises médiévales produites à Saint-Gilles du Gard. Archéologie du Midi Médiéval, 7, 1989, p. 73-106.

Marchesi 1992 : MARCHESI (H.), THIRIOT (J.), VALLAURI (L.). - Marseille Sainte-Barbe, vestiges médiévaux et modernes. Rapport de fouille de sauvetage dactylographié, Aix-en-Provence, 1992.

Pelletier 1991: PELLETIER (J.-P.), POUSSEL (L.), RIGOIR (Y. et J.) et FOY (D.), avec les contributions de AUDOIN-ROUZEAU (F.) et BRIEN-POITEVIN (F.). Poterie, métallurgie et verrerie au début du VI ${ }^{\complement}$ siècle à Gardanne (B.-du-Rh.). Documents d'Archéologie Méridionale, 14, 1991, p. 277350.

Pelletier (à paraître) : PELLETIER (J.-P.), PICON (M.), RIGOIR (Y. et J.), VALLAURI (L.). - Les productions de poteries de l'aire marseillaise et du pays d'Apt au cours de l'Antiquité tardive et du Haut Moyen Age, Actes du colloque International sur la céramique en Méditerranée, Rabat, novembre 1991.

Picon 1973 : PICON (M.). - Introduction à l'étude technique des céramiques sigillées de Lezoux. Dijon, 1973, 119 p. (Centre de recherches sur les techniques gréco-romaines).

Vasseur 1907 : VASSEUR(M.). - Sur une variété de céramique estampée. Bulletin de la Société Archéologique de Provence, $\mathrm{n}^{\circ} 8,1907$, p. 26-32.

Werner 1980 : WERNER (J.). - Arabaldo (Haribaldus) : Ein merowingischer Vir Inluster aus der Provence? (Bemerkung zu den Silbertellern von Valdonne, Dép. Bouches-du-Rhône). Mélanges de numismatique, d'archéologie et d'histoire offerts à Jean Lafaurie, Paris, 1980, p. 257-263 et pl. XXVI-XXVII. 